\title{
Effects of manual chest compression and descompression maneuver on lung volumes, capnography and pulse oximetry in patients receiving mechanical ventilation
}

Efeitos da manobra de compressão e descompressão torácica nos volumes pulmonares, capnografia e oximetria de pulso em pacientes submetidos à ventilação mecânica

Fabiana Della Via', Rosmari A. R. A. Oliveira², Desanka Dragosavac ${ }^{3}$

\begin{abstract}
Objectives: The aims of this study were to evaluate whether there are changes in lung volumes, capnography, pulse oximetry and hemodynamic parameters associated with manual chest compression-decompression maneuver (MCCD) in patients undergoing mechanical ventilation (MV). Method: A prospective study of 65 patients undergoing to MV after 24 hours. All patients received bronchial hygiene maneuvers and after 30 minutes they were submitted to ten repetitions of the MCCD during 10 consecutive respiratory cycles in the right hemithorax and than in the left hemithorax. The data were collected before the application of the maneuver and after 1, 5, 10, 15, 20, 25, 30,35 and 40 minutes following application of the maneuver. Results: There were statistical significant $(p<0.0001)$ improvements in the following parameters after MCCD maneuver during all phases of data collection until 40 minutes: inspiratory tidal volume (baseline: $458.2 \pm 132.1 \mathrm{~mL}$; post $1 \mathrm{~min}: 557.3 \pm 139.1$; post $40 \mathrm{~min}: 574.4 \pm 151$ ), minute volume (baseline: $7.0 \pm 2.7 \mathrm{~L} / \mathrm{min}$; post $1 \mathrm{~min}: 8.7 \pm 3.3$; post $40 \mathrm{~min}: 8.8 \pm 3.8$ ), and pulse oximetry (baseline: $97.4 \pm 2.2 \%$; post $1 \mathrm{~min}: 97.9 \pm 1,8$; post $40 \mathrm{~min}: 98.2 \pm 1.6 ; p<0.05$ ). There was a reduction in $\mathrm{CO}_{2}$ expired (baseline: $35.1 \pm 9.0 \mathrm{mmHg}$; post $1 \mathrm{~min}: 31.5 \pm 8.2$; post $40 \mathrm{~min}: 31.5 \pm 8.29 ; p<0.0001$ ). There was no statically significant changes in heart rate (baseline: $94.5 \pm 20.5 \mathrm{mmHg}$; post 1 min: $94.7 \pm 20.5 ;$ post 40 min: 94.92 $\pm 20.20 ; p=1$ ) and mean arterial pressure (baseline: $91.2 \pm 19.1$ bpm; post $1 \mathrm{~min}: 89.5 \pm 17.7$; post $40 \mathrm{~min}: 89.01 \pm 16.88 ; p=0.99$ ). The variables were presented in terms of means and standard deviations. Conclusion: The MCCD maneuver had positive effects by increasing lung volume and pulse oximetry and reducing $\mathrm{CO}_{2}$ expired, without promoting hemodynamic changes in patients undergoing mechanical ventilation. Trial registration RBR-268hqh.
\end{abstract}

Keywords: manual chest maneuver; mechanical ventilation; atelectasis; physical therapy.

\section{Resumo}

Objetivos: Avaliar a presença de alterações nos volumes pulmonares, oximetria de pulso, capnografia e alterações hemodinâmicas associadas à intervenção da manobra de compressão e descompressão torácica (MCDT) nos pacientes submetidos à ventilação mecânica (VM). Método: Tratou-se de um estudo prospectivo em que foram incluídos 65 pacientes em VM há mais de 24 horas. O protocolo consistiu na aplicação de manobras de higiene brônquica e, após 30 minutos, os pacientes eram submetidos a dez repetições da MCDT em dez respirações consecutivas no hemitórax direito e, posteriormente, no hemitórax esquerdo, coletando os dados antes e após a aplicação da manobra nos tempos 1, 5, 10, 15, 20, 25, 30, 35 e 40 minutos. Resultados: Constatou-se aumento significante $(p<0,001)$ do volume corrente inspiratório

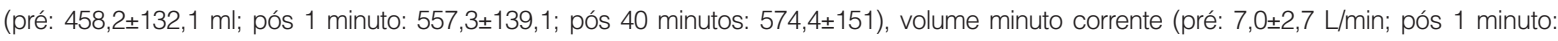

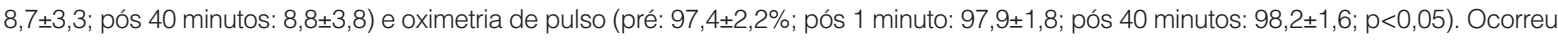

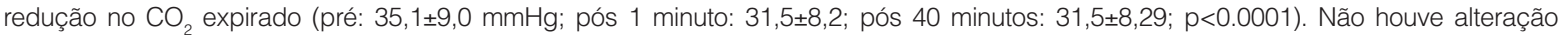
significante da frequência cardíaca (pré: $94,5 \pm 20,5$ mmHg; pós 1 minuto: $94,7 \pm 20,5$ e pós 40 minutos: 94,9 920,2 ; $p=1$ ) e pressão arterial

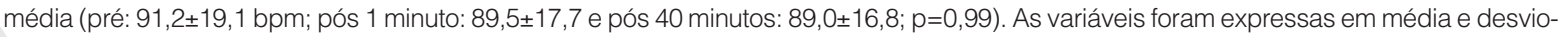
padrão. Conclusão: A MCDT possibilita a otimização dos volumes pulmonares, da oximetria de pulso e a redução do $\mathrm{CO}_{2}$ expirado sem promover alterações hemodinâmicas significantes nos pacientes submetidos à ventilação mecânica.

Registro de ensaio clínico RBR-268hqh.

Palavras-chave: manobra de compressão manual; ventilação mecânica; atelectasias; fisioterapia.

Received: 11/04/2010 - Revised: 03/23/2011 - Accepted: 09/20/2011 


\section{Introduction $: \because$.}

Hospitalized patients in intensive care units (ICU) are prone to developing respiratory complications, particularly atelectasis and bronchopneumonia ${ }^{1-5}$. The high incidence of these complications has been described. Maa et al. ${ }^{2}$ found lobular atelectasis in $82.6 \%$ of patients, and Raoof et al. ${ }^{4}$ reported a rate of $74 \%$ in patients with spinal cord injury, $85 \%$ in patients with neuromuscular disorders, over $90 \%$ of postoperative cardiac surgery patients and $20-30 \%$ postoperative abdominal surgery patients.

Hypoventilation occurs in critically ill patients as a result of the patient's inability to take deep breaths, mainly in the postoperative period following major surgery in patients with neuromuscular disorders and in those patients who have suffered major trauma that resulted in prolonged periods of immobilization ${ }^{6-11}$. Hypoventilation may also be a consequence of pain, anesthesia and sedation effects or may result from the patient's receiving mechanical ventilation, which is often poorly adjusted using inappropriate parameters $^{7}$. All together, these factors result in a progressive reduction in the functional residual capacity while the patient is under general anesthesia and in the first 48 hours following a major surgery. Diminished functional residual capacity is associated with alveolar collapse, most commonly in basal or dependent portions of the lung, making ventilation weaning more difficult and resulting in excess morbidity and mortality ${ }^{2,11}$. Since perfusion remains unaltered, an imbalance occurs in the ventilation/perfusion ratio (V/Q ratio), resulting in arterial hypoxemia ${ }^{4,7,8,11}$.

To reverse this situation or to prevent the development of atelectasis in patients receiving mechanical ventilation, re-expansion techniques such as manual hyperinsuflation ${ }^{2,12}$, high airway pressure ${ }^{13,14}$, bronchoscopy ${ }^{1}$ and manual chest compression and decompression had been used ${ }^{15}$.

Manual chest compression and decompression maneuver (MCCD) is carried out in the intensive care unit exclusively by physical therapists. This maneuver is cheaper and less aggressive than a bronchoscopy. MCCD maneuver does not require high airways pressure, reducing therefore the risk of disruption of the alveolocapillary barrier or barotrauma ${ }^{14}$, and is not necessary to disconnect the system during the MCCD maneuver, avoiding episodes of hypoxemia and the use of high fraction of inspired $\mathrm{O}_{2}$.

According to the description published by Costa $^{16}$ in the national literature, this technique consists of compressing the ribcage during the expiratory phase and maintaining the position until the initial third of the inspiratory phase when the compression is then abruptly released. Changing the chest pressure in the area of the maneuver application displaces the air, thereby expanding the chest. This maneuver can be performed during spontaneous breathing or during mechanical ventilation ${ }^{15}$.

Previous evidence ${ }^{7,16}$ suggests that the main physiological effects of MCCD maneuver are atelectatic alveolar recruitment and an increase in functional residual capacity. The use of larger tidal volume than those used by the mechanical ventilation in some respiratory cycles promote an improvement in the ventilation/perfusion ratio and a reduction in the shunt, improving oxygenation and reducing the respiratory effort, without affecting hemodynamic ${ }^{1}$.

To our knowledge no study has been published in the literature demonstrating the efficacy of this therapy. Therefore, the objectives of the present study were to evaluate the changes in pulmonary volume, oximetry and expiratory $\mathrm{CO}_{2}$ that occur following the use of MCCD maneuver in patients undergoing mechanical ventilation (MV) and the possible hemodynamic alterations (mean arterial pressure and heart rate).

\section{Method::}

The protocol of this prospective study was approved by the Internal Ethical Committee of the Pontifícia Universidade Católica de Campinas (PUCCAMP), Campinas, SP, Brazil, approval number 338/2008. The family members of sixty-five patients undergoing MV from August 2008 to January 2009 gave a written consent, accepting to participate in this study.

Inclusion criteria consisted of the following: age $\geq 18$ years, patients on mechanical ventilation, Ramsay sedation scale between 4 and 6 levels, hemodynamic stability and no osteoarticular lesions (rib fractures or unstable thorax). Exclusion criteria consisted of: intracranial hypertension $>30 \mathrm{mmHg}$, pneumothorax and hemodynamic instability (mean arterial pressure $<80 \mathrm{mmHg}$ ) with use of high doses of vasoactive drugs (dopamine and dobutamine $>5 \mu \mathrm{g} / \mathrm{kg} / \mathrm{min}$ and noradrenalin $>0.5 \mu \mathrm{g} / \mathrm{kg} / \mathrm{min}$ ).

After 24 hours of mechanical ventilation the patients were positioned in supine with the head of the bed elevated to an angle of $30^{\circ}$ and than a chest radiograph was carried out, where the radiological alteration was diagnosed by the radiologist from the hospital.

Thereafter they were submitted to bronchial hygiene therapy according to the following protocol: manual chest compression and vibrations in the expiratory phase of respiratory cycle in the previous chest wall during 10 minutes, and 
endotracheal suctioning with open system following to the instructions of the American Association of Respiratory Care ${ }^{17}$.

After 30 minutes of bronchial hygiene therapy; it was measured the first data collection prior to the maneuver and than 20 repetitions of MCCD were performed in 10 consecutive respiratory cycles in the right hemithorax and 10 repetitions in the left hemithorax. The variables were collected at 1,5 , $10,15,20,25,30,35$ and 40 minutes following the procedure. Importantly, each of these data collection occurred during spontaneous respiratory cycles.

Merely one physical therapist performed all procedures throughout the study to avoid differences in the protocol. The parameters of mechanical ventilation were maintained until the last recorded data (40 minutes after MCCD maneuver).

The following variables were monitored: inspiratory tidal volume, expiratory tidal volume, minute volume, alveolar tidal volume, alveolar minute volume, plateau pressure, peak pressure and peripheral oxygen saturation $\left(\mathrm{SpO}_{2}\right)$, expired carbon dioxide $\left(\mathrm{CO}_{2}\right)$, respiratory rate, heart rate and mean arterial pressure.

Patients were ventilated with Inter 5 plus ventilator (Intermed). Win Trace software version 3.3 Beta (Intermed) was used to measure the variables. Vital signs (pulse oximetry, heart rate and mean arterial pressure) were collected by a no invasive method using the multiparametric monitor model DX2021 ${ }^{\mathrm{TM}}$ (Dixtal) and it was used capnography module for expired $\mathrm{CO}_{2}$ (Dixtal). Before starting the study, calibration and preventive maintenance on the ventilator and monitor were performed.

The software IBM SPSS Statistics 19 was used for the statistical analysis. The clinical significance of the effect of MCCD was reported as 95\% confidence intervals (95\% CI). Post hoc analysis was performed. A repeated-measures analysis of variance (ANOVA) was performed to compare scores over time in the group on each of the depedent variables measured at each of the eleven time points: baseline, post, 1, 5, 10, 15, 20, 25, 30, 35 and 40 minutes of the study. The quantitative veriables were presented in terms of means and standard deviations. The significance level adopted was $5 \%$.

\section{Results $: \because$.}

Initially, 82 patients were included in this study; of those 17 patients were excluded from the study because they have not completed 24 hours of mechanical ventilation. Sixty-five patients receiving mechanical with Ramsay sedation scale between 4 and 6 level completed the study ( $\mathrm{n}=65)$, being $60 \%$ were male and $40 \%$ female. The mean age was 53.3 years (SD 17.5; median 54 years; range 18-92 years) (Table 1).

Abdominal surgery were the cause of hospitalization in the intensive care unit in $30.76 \%$ of the cases, neurological pathologies in $30.76 \%$ of the cases, pulmonary in $18.46 \%$, head and neck surgery in $6.15 \%$, cardiac pathologies in $6.15 \%$, orthopedic in $3.07 \%$ and vascular causes in $1.54 \%$ of the cases.

Patients were submitted to chest radiography to verify the existence of any radiological alteration (for example increased infiltration or increase opacity) prior to the maneuvers. Radiological alterations were found in $55.3 \%$ of the patients, in which $41.6 \%$ had increased infiltration in pulmonary bases, $25 \%$ atelectasis, $16.6 \%$ increased infiltration in whole lung, $11.1 \%$ emphysema signs and $5.55 \%$ neoplasia. Atelectasis had been reported in $55.55 \%$ of patients after abdominal surgery, 33.33\% with neurologic problems (strokes, subdural hematomas, intracranial hemorrhage and head injury) and $11.11 \%$ cardiac diseases (Table 1).

Table 1. Demographic data of patients ( $n=65)$.

\begin{tabular}{lc}
\hline Variables & Results \\
\hline Gender (M/F) & $60 \% / 40 \%$ \\
\hline Age (years) & $53.3 \pm 17.5^{*}$ \\
\hline Cause of hospitalization & \\
\hline Abdominal surgery & $33.8 \%$ \\
\hline Neurological pathologies & $30.8 \%$ \\
\hline Pulmonary pathologies & $18.5 \%$ \\
\hline Head and neck surgery & $6.2 \%$ \\
\hline Cardiac pathologies & $6.2 \%$ \\
\hline Orthopedic & $3.0 \%$ \\
\hline Vascular & $1.5 \%$ \\
\hline Chest radiography & \\
\hline Infiltration in bases & $41.7 \%$ \\
\hline Atelectasis & $25 \%$ \\
\hline Infiltration in whole lung & $16.7 \%$ \\
\hline Ehphysema signs & $11.1 \%$ \\
\hline Neoplasia (<3 cm) & $5.5 \%$ \\
\hline Ventilation parameters & \\
\hline PEEP & $6.0 \pm 1.9 \mathrm{cmH}_{2} 0^{*}$ \\
\hline FiO & $44.4 \pm 13.6^{*}$ \\
\hline RR & $11.2 \pm 2.8^{*}$ \\
\hline Plateau pressure & $17.9 \pm 6.9^{*}$ \\
\hline Peak pressure & $21.8 \pm 7.7^{*}$ \\
\hline SIMV/VC & $92.3 \%$ \\
\hline SIMV/PC & $7.6 \%$ \\
\hline
\end{tabular}

PEEP: Positive end-expiratory pressure; $\mathrm{FiO}_{2}$ : inspired fraction of oxygen; RR: Respiratory rate; SIMV: Synchronized intermittent mandatory ventilation; VC: Volume controlled; PC: pressure controlled ventilation. *Results expressed in mean \pm standard deviation **Respiratory rate set in ventilator. 
Table 2. Respiratory variables (mean $\pm S D)(n=65)$.

\begin{tabular}{lccccc}
\hline Time & $\begin{array}{c}\text { Inspiratory tidal } \\
\text { volume } \\
\mathrm{mL}\end{array}$ & $\begin{array}{c}\text { Expiratory tidal } \\
\text { volume } \\
\mathrm{mL}\end{array}$ & $\begin{array}{c}\text { Minute volume } \\
\mathrm{L} / \mathrm{min}\end{array}$ & $\begin{array}{c}\mathrm{CO}_{2} \\
\mathrm{mmHg}\end{array}$ & $\begin{array}{c}\mathrm{SpO} \mathrm{O}_{2} \\
\%\end{array}$ \\
\hline Baseline & $458.2 \pm 132.1$ & $457.7 \pm 155.2$ & $7.09 \pm 2.7$ & $35.1 \pm 9.0$ & $97.4 \pm 2.2$ \\
\hline Post & $557.3 \pm 139.1$ & $491.1 \pm 174.1$ & $8.41 \pm 2.7$ & $31.5 \pm 8.2$ & $97.9 \pm 1.8$ \\
\hline Post 1min & $563.7 \pm 138.4$ & $507.5 \pm 184.1$ & $8.77 \pm 3.3$ & $31.6 \pm 8.1$ & $97.9 \pm 1.7$ \\
\hline 40 min & $574.4 \pm 151.0$ & $529.9 \pm 160.7$ & $8.84 \pm 3.8$ & $31.5 \pm 8.2$ & $98.2 \pm 1.6$ \\
\hline $95 \% \mathrm{Cl}$ & 71.5 to 160.8 & 71.5 to 160.8 & 1.2 to 3.7 & 2.4 to 4.7 & $<.5$ to 1.1 \\
\hline p-value & $<0.0001$ & $<0.08 / 0.001^{*}$ & $<0.0001$ & $<0.0001$ & $<0.05$ \\
\hline
\end{tabular}

*Baseline/1 min post maneuver $p=0.08$; Baseline/40 min post maneuver $p<0.001 . \mathrm{CO}_{2}$ : Partial pressure of expired carbon dioxide; Sp0²: Peripheral oxygen saturation.

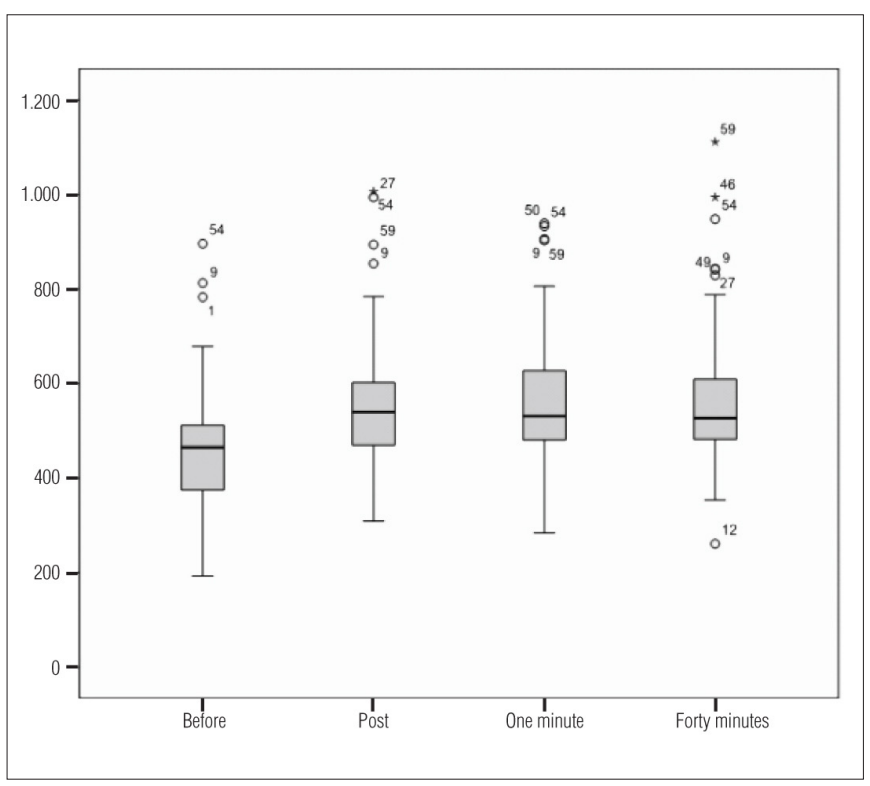

Figure 1. Variation in inspiratory tidal volume $(\mathrm{mL})$ following manual chest compression and descompression.

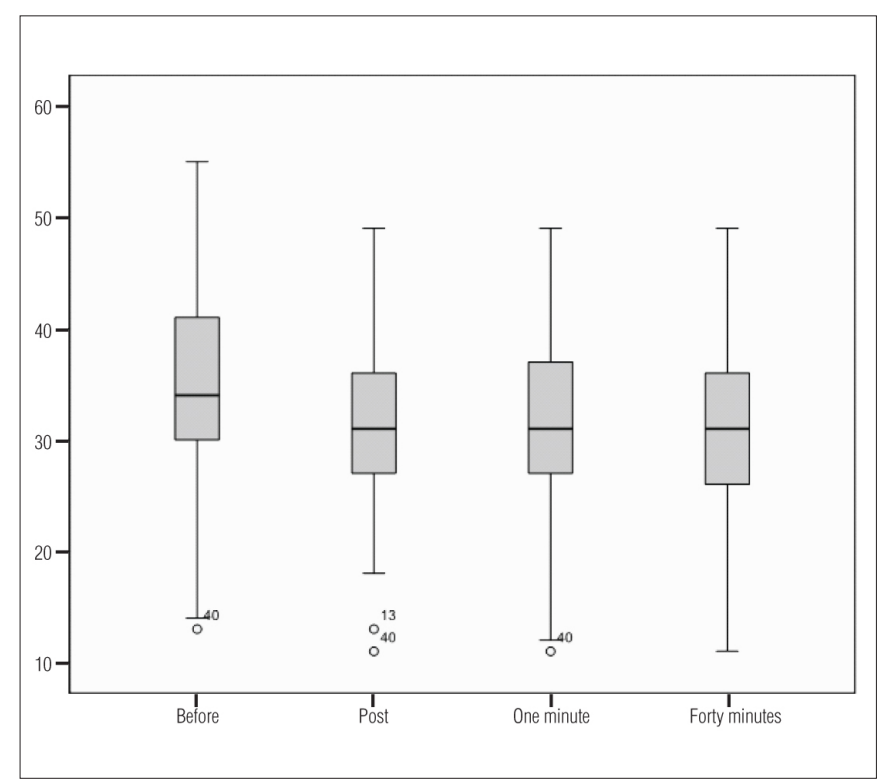

Figure 2. Variation in expired $\mathrm{CO}_{2}$ following manual chest compression and descompression.
The volume controlled synchronized intermittent mandatory ventilation (SIMV) were used in $92.3 \%$ of patients, while the remaining patients received pressure-controlled ventilation.

A prolonged increase in inspiratory tidal volume, minute volume, and expiratory tidal volume after the MCCD maneuver were found $(p<0.0001)$ (Table 2, Figure 1). When the patients were classified in two groups: group one with radiological alterations and group two without radiological alterations, in both cases the MCCD incresead the tidal volume $(\mathrm{p}<0.05)$.

A statistical significant difference was observed following manual chest compression and descompression in pulse oximetry $(\mathrm{p}<0.05)$. Capnography also revealed a reduction post and 40 minutes after the maneuver (Table 2). Moreover, capnography failed to return to baseline levels even after 40 minutes (Figure 2).

No improvements were found for the outcomes: respiratory rate

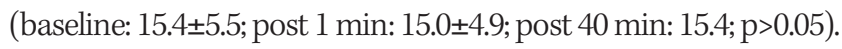

No statistically significant differences were found for the outcomes: heart rate (baseline: $94.5 \pm 20.5 \mathrm{bpm}$; post $1 \mathrm{~min}$ : $94.7 \pm 20.5$; post $40 \mathrm{~min}: 94.92 \pm 20.20 ; \mathrm{p}=1$ ) and mean arterial pressure (baseline: $91.2 \pm 19.1 \mathrm{mmHg}$; post $1 \mathrm{~min}$ : $89.5 \pm 17.7$; post 40 min: $89.01 \pm 16.88 ; p=0.99$ ), indicating that is safe to use manual chest compression in patients on mechanical ventilation.

\section{Discussion $\because \therefore$.}

Respiratory complications such as atelectasia and bronchopneumonia are common in patients receiving mechanical ventilation ${ }^{2}$. In the present study, radiological alterations were found in the chest radiograph in $55.3 \%$ of patients before the MCCD, being $25 \%$ of patients had atelectasis and it has been reported in $55.55 \%$ of patients after abdominal surgery, $33.33 \%$ with neurologic problems and $11.11 \%$ cardiac diseases. This high rate of complications has already been described in various studies ${ }^{2,4}$. 
Some studies indicate that patients undergoing abdominal surgery commonly have reductions in lung volumes, elevation of both hemidiaphragms and areas of atelectasis because of anaesthesia and monotonous ventilation ${ }^{11,18,19}$. The investigation of 22 patients undergoing abdominal surgery we found a small reduction but non-statistically significant in tidal volume.

According to Richardson and Sabanathan ${ }^{20}$ the incidence of pulmonary complications is higher after upper abdominal or chest surgery than in surgeries in other parts of the body, like in the literature; the incidence of complication (atelectasis) in abdominal surgery in this study is higher than the other surgery.

The presence of atelectasis increases the risk of pulmonary infection ${ }^{11,12}$ and is extremely important that appropriate prophylaxis should be implemented to prevent the occurrence of atelectasis or, if already present, treatment with recruitment maneuvers be initiated as early as possible.

There are controversies concerning the best way of lung re-expansion for prophylaxis and treatment of atelectasis $^{21,22}$. In the literature ${ }^{23,24}$, there are some evidence of the value of chest physical therapy in the prevention and treatment, but to our knowledge, there were no studies which examined the hemodynamic and pulmonary effects of MCCD maneuver in patients undergoing mechanical ventilation.

It is now well established that the reduction in inspiratory tidal volume persists for various days after a surgical procedure and that it is not completely reversed even when the pain is eradicated with the subarachnoid administration of opioids ${ }^{25}$. According to the literature ${ }^{25}$ the present study showed that pulmonary function could be deteriorated in patients on mechanical ventilation, mainly with regards to pulmonary volume.

According to the literature ${ }^{15}$, manual chest compression and decompression permits an increase in pulmonary tidal volume as a consequence of an increased elastic forces in the respiratory system (mainly in the ribcage) causing greater negative pleural pressure and thus, increased transpulmonary pressure, generating a greater pressure differential with an increase in flow and, consequently, greater inspired volume.

The analysis of the present study showed a statistically significant and prolonged improvement in the following parameters after manual chest compression maneuver: inspiratory tidal volume and minute volume in patients with Ramsay sedation scale between 4 and 6 levels and undergoing to synchronized intermittent mandatory ventilation without any need for positive end-expiratory pressure or hyperventilation by increasing respiratory rate or inspiratory tidal volume of the mechanical ventilator.

Studies carried out by Maa et al. ${ }^{2}$ and Stiller ${ }^{26}$ showed that offering pulmonary volumes greater than those used by the mechanical ventilation might increase transpulmonary pressure and facilitate the reopening of collapsed units, leading to an increase in ventilation and oxygenation and a decrease in $\mathrm{CO}_{2}$.

In the present study, a statistically significant difference in pulse oximetry was found following manual chest compression. A significant reduction was detected by capnography immediately following the maneuver and again 40 minutes later. In addition, capnography finds failed to return to baseline levels even after 40 minutes. These results (oxygenation) are in agreement with data published in the literature ${ }^{4}$.

Other pulmonary recruitment protocols have been described by several authors to revert atelectasis ${ }^{13,14}$. Among them, the use of consecutive and sustained hyperinsufflations with high inspiratory pressure $\left(40 \mathrm{cmH}_{2} \mathrm{O}\right)$ or increasing PEEP (Positive end-expiratory pressure) up to $15 \mathrm{cmH}_{2} \mathrm{O}$. These protocols have the potential of overdistension normal alveoli and the risk of barotraumas. In the present study the use of high inspiratory pressure or increasing PEEP was not necessary to lung re-expansion.

Sixel et al. ${ }^{27}$ conducted a study with 14 patients in mechanical ventilation with preserved ventilatory drive using the unilateral chest compression to induce expansion of the contralateral hemithorax. They concluded that unilateral elastic load application promotes reduction on tidal volume and increased in respiratory rate. When the present study is compared to the Sixel et al. ${ }^{27}$, despite the differences in the study design (Ramsay sedation scale and the application the MCCD maneuver in the same hemithorax to induce expansion), the elastic forces increased the tidal volume and maintained the respiratory rate.

According to Singer et al. ${ }^{28}$ the manual hyperinflation of critically ill patients, a re-expansion technique that improves lung volume as the MCCD maneuver produced significant reductions in cardiac output, these falls in output were directly related to the degree of hyperinflation produced rather than the increase in peak inspiratory pressure generated. Large increases in intrathoracic volume impede venous return, resulting in falls in ventricular preload and, often, cardiac output. Application of MCCD maneuver by physical therapists was found to have no statistical significant effects on hemodynamic variables (such as heart rate and mean blood pressure) in this study, indicating that 
manual chest compression may be safe to be performed in patients on mechanical ventilation.

\section{Limitations $: \because$.}

There was no control group without treatment for atelectasis, because we considered such protocol unethical. Each patient was his/her own control. The other limitation in the present study is that chest radiograph and arterial blood analysis were not performed in all patients after the MCCD maneuver; therefore, there was no comparison of these data with the data prior to the maneuver.

\section{Conclusion $: \because$.}

Manual chest compression was useful in mechanically ventilated patients. The MCCD maneuver increased lung volumes and pulse oximetry and reduced expiratory $\mathrm{CO}_{2}$, without hemodynamic alterations in patients submitted to mechanical ventilation.

\section{References $: \because$.}

1. Kreider ME, Lipson DA. Bronchoscopy for atelectasis in the ICU: a case report and review of the literature. Chest. 2003;124(1):344-50.

2. Maa SH, Hung TJ, Hsu KH, Hsieh YI, Wang KY, Wang CH, et al. Manual hyperinflation improves alveolar recruitment in difficult-to-wean patients. Chest. 2005;128(4):2714-21.

3. Unoki T, Kawasaki Y, Mizutani T, Fujino Y, Yanagisawa Y, Ishimatsu S, et al. Effects of expiratory rib-cage compression on oxygenation, ventilation, and airway-secretion removal in patients receiving mechanical ventilation. Respir Care. 2005;50(11):1430-7.

4. Raoof S, Chowdhrey N, Raoof S, Feuerman M, King A, Sriraman R, et al. Effect of combined kinetic therapy and percussion therapy on the resolution of atelectasis in critically ill patients. Chest. 1999;115(6):1658-66.

5. Tweed WA, Phua WT, Chong KY, Lim E, Lee TL. Tidal volume, lung hyperinflation and arterial oxygenation during general anaesthesia. Anaesth Intensive Care. 1993;21(6):806-10.

6. Kravitz RM. Airway clearance in Duchenne Muscular Dystrophy. Pediatrics. 2009;123(Suppl 4):S231-5.

7. Scanlan C, Wilkins R, Stoller J. Fundamentos da terapia respiratória de Egan. São Paulo: Manole; 2000

8. Rezaiguia S, Jayr C. [Prevention of respiratory complications after abdominal surgery]. Ann $\mathrm{Fr}$ Anesth Reanim. 1996;15(5):623-46.

9. Lindberg P, Gunnarson L, Tokics L, Secher E, Lundquist H, Brismar B, et al. Atelectasis and lung function in the postoperative period. Acta Anaesthesiol Scand. 1992;36(6):546-53.

10. Woodring JH, Reed JC. Types and mechanisms of pulmonary atelectasis. J Thorac Imaging. 1996;11(2):92-108.

11. Siafakas N, Mitrouska I, Bouros D, Georgopoulos D. Surgery and the respiratory muscles. Thorax. 1999;54(5):458-65.

12. Stiller K, Geake T, Taylor J, Grant R, Hall B. Acute lobar atelectasis. A comparison of two chest physiotherapy regimens. Chest. 1990;98(6):1336-40.

13. Malbouisson LMS, Humberto F, Rodrigues RR, Carmona MJC, Auler Júnior JOC. Atelectasias durante anestesia: fisiopatologia e tratamento. Rev Bras Anestesiol. 2008;58(1):73-83.

14. Rothen HU, Neumann P, Berglund JE, Valtysson J, Magnusson A, Hedenstierna G. Dynamics of re-expansion of atelectasis during general anaesthesia. Br J Anaesth. 1999;82(4):551-6.
15. Sarmento G, Veja J, Lopes N. Fisioterapia em UTI. São Paulo: Atheneu; 2006.

16. Costa D. Fisioterapia respiratória básica. São Paulo: Atheneu; 1999

17. AARC clinical practice guideline. Endotracheal suctioning of mechanically ventiladed adults and children with artificial airways. American Association for Respiratory Care. Respir Care. 1993;38(5):500-4.

18. Dureuil B, Cantineau JP, Desmonts JM. Effects of upper or lower abdominal surgery on diaphragmatic function. Br J Anaesth. 1987;59(10):1230-5.

19. Jaber S, Delay J, Chanques G, Sebbane M, Jacquet E, Souche B, et al. Outcomes of patients with acute respiratory failure after abdominal surgery treated with noninvasive positive pressure ventilation. Chest. 2005;128(4):2688-95.

20. Richardson J, Sabanathan S. Prevention of respiratory complications after abdominal surgery. Thorax. 1997;52(Suppl 3):S35-40.

21. Pasquina $P$, Tramèr MR, Granier JM, Walder B. Respiratory physiotherapy to preven pulmonary complications after abdominal surgery: a systematic review. Chest. 2006;130(6): 1887-99.

22. Johnson N, Marini JJ, Pierson DJ, Hudson LD. Acute lobar atelectasis: effect of chest percussion and postural drainage (CPPD) on resolution. Am Rev Respir Dis. 1987;135:A433.

23. Jerre G, Silva TJ, Beraldo M, Gastaldi A, Kondo C, Leme F, et al. Fisioterapia no paciente sob ventilação mecânica. III consenso Brasileiro de Ventilação Mecânica. J Bras Pneumol. 2007;33(Supl 2):S142-50.

24. Schindler MB. Treatment of atelectasis: where is the evidence? Crit Care. 2005;9(4):341-2.

25. Pflug AE, Murphy TM, Butler SH, Tucker GT. The effects of postoperative peridural analgesia on pulmonary therapy and pulmonary complications. Anesthesiology. 1974;41:8-17.

26. Stiller K. Physiotherapy in intensive care: towards an evidence-based practice. Chest 2000;118(6):1801-13.

27. Sixel BS, Lemes DA, Pereira KA, Guimarães FS. Compressão manual torácica em pacientes com insuficiência respiratória aguda. Fisioter Bras. 2007:8(2):103-6.

28. Singer M, Vermaat J, Hall G, Latter G, Patel M. Hemodynamic effects of manual hyperinflation in critically ill mechanically ventilated patients. Chest. 1994;106(4):1182-7. 